\title{
Association between Irritable Bowel Syndrome and Risk of Osteoporosis in Korean Premenopausal Women
}

\author{
Sang-Yeoup Lee ${ }^{a, b}$ Hye-Rim Hwang ${ }^{c}$ Yu-Hyeon $\mathrm{Yi}^{\mathrm{b}, \mathrm{c}}$ Jin-Mi Kim ${ }^{\mathrm{d}}$ Yun-Jin Kim ${ }^{\mathrm{b}, \mathrm{c}}$ \\ Jeong-Gyu Lee ${ }^{b, c}$ Young-Hye Cho ${ }^{a, b}$ Young-Jin Tak ${ }^{b, c}$ Seung Hun Lee ${ }^{b, c}$ Eun Ju Park ${ }^{a, b}$ \\ Youngin Lee ${ }^{a, b}$ \\ aFamily Medicine Clinic, Obesity, Metabolism and Nutrition Center, Pusan National University Yangsan Hospital, \\ Yangsan, Republic of Korea; ${ }^{b}$ Pusan National University School of Medicine, Yangsan, Republic of Korea; \\ 'Department of Family Medicine, Medical Research Institute and Busan Tobacco Control Center, Pusan National \\ University Hospital, Busan, Republic of Korea; ${ }^{\mathrm{d}}$ Department of Biostatistics, Clinical Trial Center, Biomedical Research \\ Institute, Pusan National University Hospital, Busan, Republic of Korea
}

\section{Highlights of the Study}

- Irritable bowel syndrome (IBS) and osteoporosis are both more prevalent in women than in men.

- IBS may increase the risk of osteoporosis and related fractures in women than in age-matched controls.

- After adjusting for known confounding variables, the increase in the risk of osteoporosis remained marginally significant.

\section{Keywords}

Irritable bowel syndrome · Osteoporosis - Osteoporotic fracture

\begin{abstract}
Objective: The objective of this study is to evaluate irritable bowel syndrome (IBS) as a risk factor for osteoporosis and osteoporotic fracture in Korean women after controlling for basic confounding factors and considering detailed demographic and clinical information. Subjects and Methods: We performed a nationwide population-based retrospective cohort analysis and matched every IBS case with a non-IBS case at a 1:4 frequency ratio based on age. The population consisted of female patients with data in the Health Insurance Review and Assessment (HIRA) database from 2002 to 2010. To determine the risk of osteoporosis and osteoporotic frac-
\end{abstract}

karger@karger.com www.karger.com/mpp

Karger $\stackrel{\text { ' }}{5}$
(C) 2021 The Author(s)

Published by S. Karger AG, Basel

This is an Open Access article licensed under the Creative Commons Attribution-NonCommercial-4.0 International License (CC BY-NC) (http://www.karger.com/Services/OpenAccessLicense), applicable to the online version of the article only. Usage and distribution for commercial purposes requires written permission. ture in IBS and non-IBS patients, hazard ratios (HRs) with 95\% confidence intervals $(\mathrm{Cl})$ were estimated using Cox proportional hazards regression models, adjusting for confounding variables, such as the area of residence, health insurance type, and economic status. Results: We identified $1,017,468$ patients in the HIRA database with data from 2002 to 2010 who could potentially be included in the cohort. Among these, we identified 1,545 (11.4\%) women (age >19 years) with newly diagnosed IBS (IBS group). Additionally, 6,180 patients without IBS and age-matched to the IBS group were selected. Cox modeling revealed that the crude HRs for osteoporosis and osteoporotic fractures in patients with IBS were $1.476(95 \% \mathrm{Cl}$, $1.241-1.754)$ and $1.427(95 \% \mathrm{Cl}, 1.086-1.876)$, respectively. Conclusion: Our data showed an increased incidence of osteoporosis and osteoporotic fractures in women with IBS compared with age-matched controls.

(C) 2021 The Author(s).

Published by S. Karger AG, Basel 


\section{Introduction}

Irritable bowel syndrome (IBS) and osteoporosis are more common in women than in men [1]. IBS can hinder daily activities, decrease the quality of life, and pose a social and medical burden on the patient. Altered intestinal motility, visceral hypersensitivity, psychological disturbance, or a combination of any of these conditions may be implicated in the pathogenesis of IBS [2]. Chronic psychological stress has been reported to intensify bowel irritability by triggering mucosal inflammation, which adversely affects gastrointestinal absorption [3]. Secondary osteoporosis is common in patients with gastrointestinal diseases, and its pathogenesis is multifactorial. Malabsorption, calcium and vitamin $\mathrm{D}$ deficiencies, secondary hyperparathyroidism, glucocorticoid use, hypogonadism, low body mass index (BMI), and chronic systemic inflammation are known causes of metabolic bone disease in patients with gastrointestinal diseases [4].

Osteoporosis is a growing, major public health burden, with medical and socioeconomic effects that decrease the quality and span of life [5]. Chronic inflammatory pathological conditions affecting the liver and gastrointestinal tract are commonly associated with osteopenia and osteoporosis [6]. Several other factors that increase the risk of osteoporosis are well known. Smoking is an independent risk factor for osteoporosis in postmenopausal women, and physical activity is a protective factor for bone mass retention [7].

Ulcerative colitis, Crohn's disease, and celiac disease have been previously described as augmenting the risk of osteoporosis and related fractures. A recent study found that the risk of osteoporosis was higher among patients suffering from these diseases [8]. However, the study did not evaluate the effects of medications on the risk of osteoporosis and fractures. Furthermore, detailed patient data regarding smoking habits, alcohol consumption, BMI, and physical activity were not included. Another study conducted in Taiwan reported a higher incidence of osteoporosis among patients with IBS [9], but the study design did not account for basic confounding factors such as age. Statistical matching between exposed and control groups can be used to equalize the distribution of these confounders, improving the validity of any association found.

We sought to determine whether IBS is associated with an increased risk of osteoporosis and related fractures in women. We conducted a large, retrospective, populationbased cohort study of Korean female patients with IBS and frequency-matched controls using data from the
Health Insurance Review and Assessment (HIRA) database of the National Health Insurance Service (NHIS), adjusting for known confounding risk factors (smoking, exercise habits, and BMI).

\section{Subjects and Methods}

\section{Data Sources and Study Population}

The HIRA database is the service claims database of the Korean NHIS. The NHIS is a single-payer system with mandatory registration for all residents of Korea. The HIRA database stores population-based electronic records of all medical and prescription drug claims in Korea and can be used as a source of vital patient data, such as clinical diagnoses, hospital discharge diagnoses, and details of medical management including prescriptions, investigational drugs, procedures, and operations [10]. HIRA claims data are collected when health-care service providers submit claims to be reimbursed for providing a service to a patient and are based on the major or minor diagnostic code associated with the treatment. The prescription data include brand names, dosages of drugs, duration of treatment, and assigned Anatomical Therapeutic Chemical Classification codes [11]. Information about health behavior, such as smoking status, drinking, exercise, and diet, is not included in the HIRA database, although these missing data are often as important as the outcomes, risk factors, or exposures. However, information regarding lifestyle factors, such as drinking, smoking, exercise, diet, height, weight, BMI, and blood pressure, can be made available by linking to the Korean National Health and Nutritional Survey of the Korea Center for Disease Control and Prevention [12]. The NHIS-Health Screening Cohort (NHIS-HEALS) includes participants who have completed health screening programs provided by the NHIS in the Republic of Korea. The NHIS constructed the NHIS-HEALS cohort database in 2015. In constructing the NHISHEALS database, a sample cohort was first selected from the 2002 and 2003 health screening participants aged 40-79 years in 2002 and followed up through 2013 [13]. We used the NHIS-HEALS database from insurance claims and health checkups to obtain patients' sociodemographic and clinical characteristics, including area of residence (metropolitan or not), health insurance (regional, occupational, Medicaid), age-group, economic status, smoking habits (current, ex-, or nonsmoker), history of high-risk alcohol consumption ( $>2$ times/week), history of a lack of exercise $(<1-2$ times/week), height, and weight. We used lifestyle data from the year the subject was initially selected into the cohort and did not include any changes during the follow-up period.

Patients were divided into age-groups with 5-year ranges and were assigned to lower, lower-middle, upper-middle, and upper based on income quintile levels from 0 (lowest) to 10 (highest). We excluded patients $<19$-years old male; with IBS $<6$ months prior to enrollment; with osteoporosis or osteoporotic fractures $<6$ months prior to enrollment; took medications for osteoporosis treatment $<6$ months prior to enrollment; with malignant neoplasms; and those who had undergone menopause.

This study was exempted from ethical approval requirements by the Research Ethics Board of Pusan University Hospital (E2014090, July 25, 2014). Informed consent was not required for this study because it used anonymous patient health records released by HIRA, with no personally identifiable information. 
Identification of Patients with IBS

We identified an exposed cohort consisting of patients who were newly diagnosed with IBS from January 2002 to December 2010. We excluded 10 disease categories for a more accurate IBS diagnosis, including gastrointestinal cancer, inflammatory bowel disease, and peptic ulcer disease (see online suppl. 1; for all online suppl. material, see www.karger.com/doi/10.1159/000517909). According to the International Classification of Diseases, Tenth Revision (ICD-10), there are 2 types of IBS: diarrhea-predominant (code K580) and nondiarrhea (codes K58, K589, K580 + K589 + K58, K58 + K589, or K58 + K580).

\section{Identification of Patients with Osteoporosis and Osteoporotic}

\section{Fractures}

We identified patients with osteoporosis and osteoporotic fractures primarily using ICD-10 codes; however, to improve accuracy and avoid omitting any such patients from the study, we also referred to records of drug use, surgery, or medical procedures. First, we excluded patients with diseases that could cause osteoporosis (autoimmune; ankylosing spondylitis, rheumatoid arthritis, systemic lupus, digestive, and gastrointestinal; diseases of malabsorption; coeliac disease, Crohn's disease, inflammatory bowel disease, lactose intolerance, and endocrine disorders; Cushing's syndrome, hyperparathyroidism, and diabetes; hypogonadal states; male and female; and primary or secondary; e.g., Turner syndrome/Klinefelter syndrome, amenorrhea, Kallmann syndrome, and hematologic; hemophilia, leukemia, and lymphomas, monoclonal gammopathies, multiple myeloma, sickle cell disease, systemic mastocytosis, thalassemia, and neurological; epilepsy, dementia, psychophysiological disorders, and mental illness; depression, anorexia nervosa, HIV/AIDS, amyloidosis, chronic metabolic acidosis, chronic obstructive pulmonary disease and asthma, chronic kidney disease, hypercalciuria, sarcoidosis, and congestive heart failure) [14].

Second, we excluded patients who took medications capable of inducing osteoporosis for $>6$ months (online suppl. 2) [14]. Next, we extracted the information of patients assigned any of the ICD10 codes M80 (osteoporosis with pathological fracture), M81 (osteoporosis without pathological fracture), or M82 (osteoporosis in other diseases classified elsewhere). Then, the prescriptions of patients with such codes were reviewed for osteoporosis medications (bisphosphonates, a combination of bisphosphonates and vitamin $\mathrm{D}$, selective estrogen-receptor modulators, or calcium carbonate). As a result, we identified premenopausal women in the HIRA database with ICD-10 codes for osteoporosis and medication for osteoporosis treatment without having disease and prescription codes for causes of osteoporosis.

We identified osteoporotic fracture events using particular ICD-10 codes in patients with osteoporosis, including those for vertebral (M48.4, M48.5, S120-9, S22.0, S22.1, and S32.0-8), clavicle (S42.0), humerus (S42.2 and S42.4), wrist (S52.2-9), hip (S72.0-2), and ankle (S82.3, S82.5, and S82.6) fractures [15-18]. Fractures were further identified using the ICD-10 codes for 7 operative procedures (open reduction of fractured extremity and closed pinning; femur, external fixation; pelvis or femur, closed reduction of fractured extremity; pelvis or femur, bone traction, skin traction, and hemiarthroplasty; and hip) [19].

Statistical Analysis

We performed a frequency matching of unexposed controls with patients with IBS (ratio 1:4) on the basis of age-group. We followed the IBS cohort and matched controls from the enrollment date to December 31,2010, or to the date of diagnosis of osteoporosis or an osteoporotic fracture, whichever came first. Diagnoses in the database were coded according to the ICD-10. Descriptive statistics were used to express baseline study population data, including the proportion, with percentage, for categorical variables and the mean \pm SD for continuous variables. The $\chi^{2}$ test and the Wilcoxon rank-sum test were used to characterize associations between IBS status and patient characteristics. The Kaplan-Meier method was used to estimate the risk to osteoporosis-free patients of developing osteoporosis and consequent fractures, with the logrank test used to determine whether there was a significant difference between patients with IBS and controls. Further evaluation of this risk was performed by applying Cox proportional hazards regression modeling to estimate hazard ratios (HRs) with $95 \%$ confidence intervals (CIs) for each group. In some models, HR was adjusted for available confounding variables, such as smoking, drinking, and exercise habits. As not all patients had received a health checkup prior to diagnosis, the available confounding variables for analysis were limited. All analyses were performed using SAS software, version 9.2 (SAS Institute, Inc., Cary, NC, USA). $p$ values $<0.05$ were considered a priori to be statistically significant.

\section{Results}

We identified $1,017,468$ patients with HIRA data recorded from 2002 to 2010 who could potentially be included in the cohort. After applying precise exclusion criteria as described above, we identified 1,545 patients newly diagnosed with IBS. After frequency matching based on age, 6,180 patients without IBS were selected. Baseline data for the study population are given in Table 1 . Patients in the IBS cohort were less likely to reside in metropolitan areas, but they had a higher rate of occupational insurance coverage, indicating a higher rate of employment. The rate of high-risk drinking and the proportion of underweight patients were significantly higher in the IBS group than in the control group.

Kaplan-Meier analysis found an increased cumulative risk of osteoporosis and osteoporotic fractures in patients with IBS compared to that with age-matched controls (Fig. 1). The proportion of patients who developed osteoporosis during the study period was higher in the IBS group than in the non-IBS group (11.4 vs. 7.8\%). Cox proportional hazards regression modeling found the crude HR for osteoporosis in the IBS cohort to be 1.476 (95\% CI, 1.2411.754) (Table 2). After adjusting for sociodemographic confounding variables, such as the area of residence, health insurance type, and economic status (Model 1), the HR for osteoporosis was again found to be significantly elevated in those with IBS. However, when HR was adjusted for clinical confounders, including high-risk drinking, exercise, and 
Table 1. Demographic and clinical characteristics of study subjects

\begin{tabular}{|c|c|c|c|c|}
\hline & Overall & Non-IBS & IBS & $p$ value* \\
\hline Subjects, $n$ & 7,725 & 6,180 & 1,545 & \\
\hline \multicolumn{5}{|l|}{ Age group } \\
\hline 20-39 years & $5,070(65.6)$ & $4,056(65.6)$ & $1,014(65.6)$ & \multirow{3}{*}{1.000} \\
\hline 40-64 years & $2,290(29.6)$ & $1,832(29.6)$ & $458(29.6)$ & \\
\hline 65 years or older & $365(4.7)$ & $292(4.7)$ & $73(4.7)$ & \\
\hline \multicolumn{5}{|l|}{ Area of residence } \\
\hline Metropolitan & $5,065(65.6)$ & $4,265(69.0)$ & $800(51.8)$ & \multirow{2}{*}{$<0.001$} \\
\hline Nonmetropolitan & $2,660(34.4)$ & $1,915(31.0)$ & $745(48.2)$ & \\
\hline \multicolumn{5}{|l|}{ Health insurance } \\
\hline Regional insurance & $5,620(72.8)$ & 4,919 (79.6) & $701(45.4)$ & \multirow{3}{*}{$<0.001$} \\
\hline Occupational insurance & $1,898(24.6)$ & $1,057(17.1)$ & $841(54.4)$ & \\
\hline Medicaid & $207(2.7)$ & $204(3.3)$ & $3(0.2)$ & \\
\hline \multicolumn{5}{|l|}{ Economic status } \\
\hline Lower & 1,207 (15.6) & $950(15.4)$ & $257(16.6)$ & \multirow{4}{*}{0.176} \\
\hline Lower middle & $1,997(25.9)$ & $1,597(25.8)$ & $400(25.9)$ & \\
\hline Upper middle & $2,532(32.8)$ & $2,059(33.3)$ & $476(30.6)$ & \\
\hline Upper & $1,989(25.7)$ & $1,574(25.5)$ & $415(26.9)$ & \\
\hline \multicolumn{5}{|l|}{ Smoking } \\
\hline None & 1,105 (95.6) & $692(94.8)$ & $413(96.9)$ & \multirow{3}{*}{0.194} \\
\hline Current & $40(3.5)$ & $29(4.0)$ & $11(2.6)$ & \\
\hline Past & $11(1.0)$ & $9(1.2)$ & $2(0.5)$ & \\
\hline High-risk drinking & $23(1.9)$ & $14(1.8)$ & $9(2.1)$ & 0.945 \\
\hline Exercise & $74(6.2)$ & $49(6.4)$ & $25(5.7)$ & 0.721 \\
\hline Height, cm & $157.1 \pm 6.1$ & $156.8 \pm 6.0$ & $157.6 \pm 6.1$ & 0.032 \\
\hline Weight, kg & $55.7 \pm 8.1$ & $56.3 \pm 8.4$ & $54.6 \pm 7.6$ & $<0.001$ \\
\hline $\mathrm{BMI}, \mathrm{kg} / \mathrm{m}^{2}$ & $22.3 \pm 3.2$ & $22.9 \pm 3.3$ & $22.0 \pm 3.0$ & $<0.001$ \\
\hline Underweight $\left(<18 \mathrm{~kg} / \mathrm{m}^{2}\right)$ & $146(11.8)$ & $82(10.4)$ & $64(14.4)$ & 0.045 \\
\hline
\end{tabular}

Data are presented as mean \pm standard deviation or $n(\%)$. Metropolitan areas included Seoul, Incheon, Daejeon, Gwangju, Daegu, Ulsan, and Busan. Other areas were considered nonmetropolitan. Underweight is defined as BMI $<18 \mathrm{~kg} / \mathrm{m}^{2}$. High-risk drinking refers to alcohol consumption more than twice a week with the average amount of alcohol consumed being 5 drinks or more. Exercise refers to engaging any of the following during the past week: (1) strenuous physical activity for $>20$ min a day and $>3$ days a week; (2) moderate physical activity for $>30$ min a day and >5 days a week; or (3) or more. BMI, body mass index; IBS, irritable bowel syndrome. * $p$ value between the non-IBS and IBS groups as calculated by the $x^{2}$ test or the Wilcoxon rank-sum test.

BMI, in addition to sociodemographic factors (Model 2), with only patients who had health checkup data included in the analysis, there was a marginally significant difference between the IBS and non-IBS groups.

Table 3 shows case ratios and HRs for osteoporotic fractures in the IBS and non-IBS cohorts. Cox proportional hazards regression modeling found the crude HR for osteoporotic fractures in the IBS cohort to be 1.427 (95\% CI, 1.086-1.876). After adjusting for known confounding variables as before, Model 1 showed an increased risk of osteoporotic fractures in the IBS cohort, and in Model 2, the HR reversed to 0.997 (95\% CI, $0.560,1.774)$, although it was not statistically significant.

\section{Discussion}

In this large-scale, population-based cohort study, we found that IBS was associated with an increased risk of osteoporosis in Korean women. Women with IBS had a higher incidence of osteoporosis, and their cumulative risk of osteoporosis and consequent osteoporotic fractures was also found to be elevated. However, after adjusting for known confounding variables, this significant increase in risk disappeared. The HR was analyzed by adjusting for available confounding variables, such as smoking, drinking, and exercise. Smoking and drinking were considered risk factors for osteoporosis and were used for correction [7]. However, we used lifestyle data from the year the subject was initially selected into the 
Table 2. Cox proportional hazards regression modeling of the risk of osteoporosis in women with irritable bowel syndrome

\begin{tabular}{llllll}
\hline & Patients, $n$ & Events, $n$ & $\begin{array}{l}\text { Percentage } \\
\text { with events }\end{array}$ & HR [95\% CI] & $p$ value \\
\hline $\begin{array}{l}\text { Crude } \\
\quad \text { IBS }\end{array}$ & 1,545 & 176 & 11.4 & $1.476[1.241,1.754]$ & $<0.001$ \\
$\quad \begin{array}{l}\text { Non-IBS } \\
\text { Model } 1\end{array}$ & 6,180 & 479 & 7.8 & & \\
$\quad$ IBS & 1,545 & 176 & 11.4 & $1.416[1.171,1.713]$ & $<0.001$ \\
$\quad$ Non-IBS & 6,180 & 479 & 7.8 & & \\
$\begin{array}{l}\text { Model 2 } \\
\text { IBS }\end{array}$ & 434 & 71 & 16.4 & $1.361[0.984,1.882]$ & 0.063 \\
$\quad$ Non-IBS & 754 & 111 & 14.7 & & \\
\hline
\end{tabular}

Model 1 was adjusted for area of residence, health insurance, and economic status. Model 2 was adjusted for area of residence, health insurance, economic status, high-risk drinking, exercise, and BMI. IBS and osteoporosis were defined using diagnostic codes. Patients newly diagnosed with IBS from 2003 to 2010 were 1:4 frequency-matched with the non-IBS group on the basis of age groups (5-year range per group). HR, hazard ratio; $95 \% \mathrm{Cl}, 95 \%$ confidence interval; BMI, body mass index; IBS, irritable bowel syndrome.
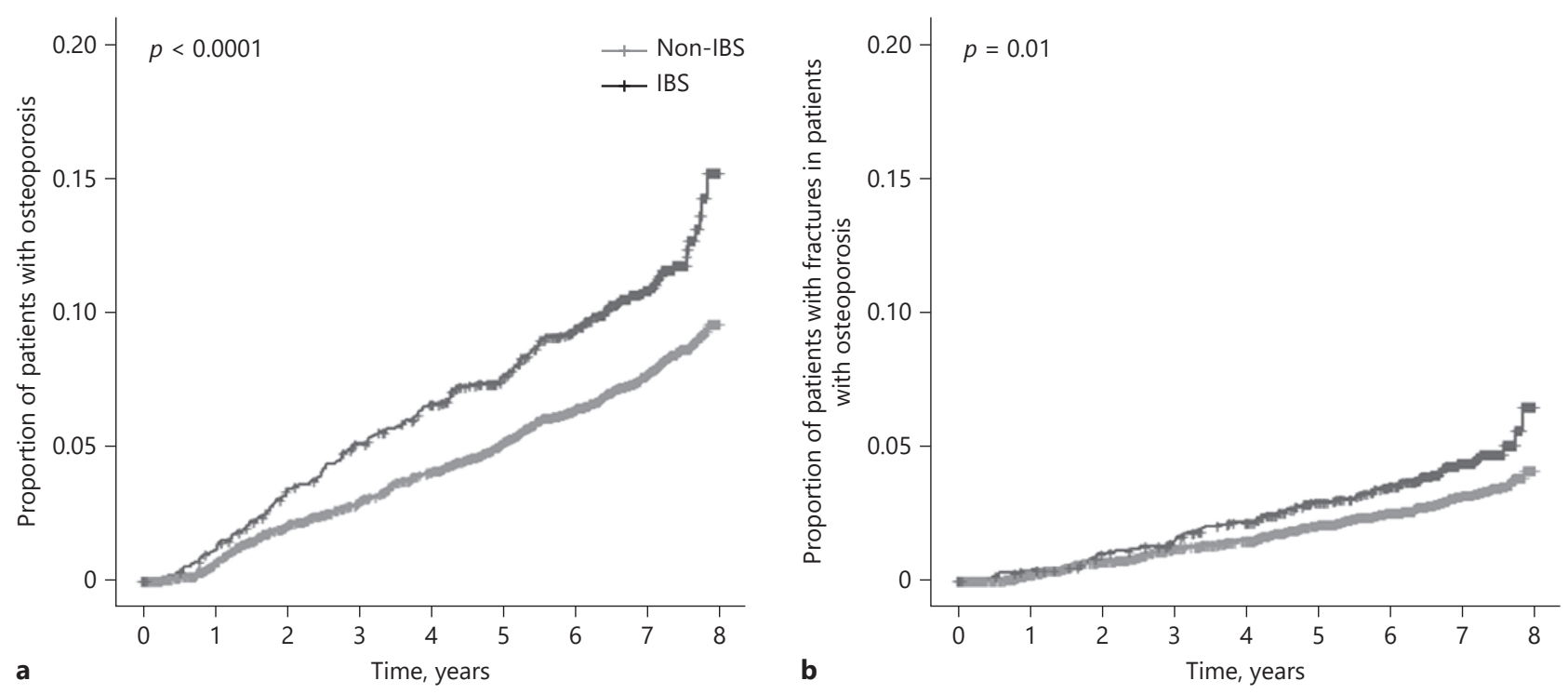

Fig. 1. The cumulative risk of osteoporosis (a) and osteoporotic fracture (b) in Korean women with and without IBS. IBS, irritable bowel syndrome.

cohort and did not include any changes during the follow-up period. Thus, there may be a potential bias in the lifestyle factors used for correction. As all subjects had not received a prior health checkup, the available confounding variables used for the analysis were limited. In the analysis to adjust for high-risk drinking, exercise, and $\mathrm{BMI}$, the number of subjects was drastically reduced, resulting in a bias for selection.
Previous epidemiological studies have examined the association between IBS and the risk of osteoporosis. Some of our results were similar to those reported by Stobaugh et al. [8] in the USA. They used the Nationwide Emergency Department Sample to review the records of 317,857 emergency department visits by patients with secondary IBS diagnoses. Within the assessed group, 17,752 patients were diagnosed with osteoporosis, and 2,197 of 
Table 3. Cox proportional hazards regression modeling of the risk of osteoporotic fracture in women with irritable bowel syndrome

\begin{tabular}{llllll}
\hline & Patients, $n$ & Events, $n$ & $\begin{array}{l}\text { Percentage } \\
\text { with events }\end{array}$ & HR [95\% CI] & $p$ value \\
\hline $\begin{array}{l}\text { Crude } \\
\quad \text { IBS }\end{array}$ & 1,545 & 70 & 4.5 & $1.427[1.086,1.876]$ & 0.011 \\
$\quad \begin{array}{l}\text { Non-IBS } \\
\text { Model } 1\end{array}$ & 6,180 & 195 & 3.2 & & \\
$\quad$ IBS & 1,545 & 70 & 4.5 & $1.302[0.967,1.753]$ & 0.082 \\
$\quad$ Non-IBS & 6,180 & 195 & 3.2 & & \\
$\begin{array}{l}\text { Model 2 } \\
\text { IBS }\end{array}$ & 434 & 20 & 4.6 & $0.997[0.560,1.774]$ & 0.992 \\
$\quad$ Non-IBS & 754 & 38 & 5.0 & & \\
\hline
\end{tabular}

Model 1 was adjusted for area of residence, health insurance, and economic status. Model 2 was adjusted for area of residence, health insurance, economic status, high-risk drinking, exercise, and BMI. IBS and osteoporosis were defined using diagnostic codes. Patients newly diagnosed with IBS from 2003 to 2010 were 1:4 frequency-matched with the non-IBS group on the basis of age groups (5-year range per group). $\mathrm{HR}$, hazard ratio; $95 \% \mathrm{Cl}, 95 \%$ confidence interval; BMI, body mass index; IBS, irritable bowel syndrome. these patients had a concurrent fracture diagnosis. The researchers found that the risk of osteoporosis was higher among patients who had IBS than among those who did not (adjusted odds ratio, 4.28; 95\% CI, 4.21-4.35), as was the risk of osteoporotic fractures (odds ratio, $2.36 ; 95 \% \mathrm{CI}$, 2.26-2.47). However, their data did not account for the potential effects of medications on the risk of osteoporosis and consequent fractures. Another study, conducted in Taiwan, also showed results similar to ours, finding a higher incidence of osteoporosis among patients with IBS than among those without IBS (HR 1.65; 95\% CI, 1.541.77). In the Taiwanese study, female patients aged 40-59 years who had IBS had a higher risk of developing osteoporosis than women in the same age range who did not have IBS (HR, 4.42; 95\% CI, 3.37-5.79) [9]. However, the Taiwanese data did not account for the potential effects of weight or drinking habits on the risk of osteoporosis and consequent fractures. In our study, 1:4 frequency matching for age was performed to neutralize differences in IBS prevalence by age, and exclusion criteria were designed to rule out patients who had non-IBS diseases or other conditions associated with osteoporosis and fractures at baseline. By applying a precise and comprehensive research design using a Kaplan-Meier analysis, we found that the cumulative risk of osteoporosis might be increased in women with IBS and that their risk of osteoporotic fracture was significantly higher. We also found statistically significant increases in the HR for osteoporosis among patients with IBS; however, because we did not have data on confounding clinical variables for all our subjects, we were able to adjust for these variables only in limited cases.
In previous epidemiological studies, osteoporotic fractures were defined as follows: diagnosis of osteoporosis with or without pathological fracture (ICD-10 code M80.* or M81.*) or at least one prescription of a potentially osteoporosis-related medication (calcium or calcium and vitamin $\mathrm{D}$, hormone therapy, bisphosphonates, raloxifene, teriparatide, and fluoride), and diagnosis of an osteoporotic fracture (S22.*-S82.*) [16-18]. In this study, vertebral fractures (M48.4, M48.5, S120-9) and 7 operative procedures (open reduction of fractured extremity and closed pinning; femur and external fixation; pelvis or femur and closed reduction of fractured extremity; pelvis or femur, bone traction, skin traction, and hemiarthroplasty; and hip) were additionally included for the operational definitions of osteoporotic fractures [19]. Fracture incidence may have been overestimated in this study because it included a wider range of fracture codes and surgical codes than in previous studies. The new method used this time to define osteoporotic fractures needs to be further validated.

Abraham et al. [20] reported that in adults with inflammatory bowel disease, the risk of low bone mineral density was significantly increased by vitamin $\mathrm{D}$ deficiency, female sex, Asian ethnicity, Crohn's disease, and corticosteroid use. In our study, patients with IBS were identified based on the ICD-10 code rather than by colonoscopy results or Rome diagnostic criteria. To overcome this shortcoming and improve the accuracy of our results, we excluded 10 disease categories that may cause similar symptoms, including gastrointestinal cancer, inflammatory bowel disease, and peptic ulcer disease (online suppl. 
1). There are a number of factors that may be associated with the development of osteoporosis; therefore, it is necessary to account for them when assessing the independent effects of IBS. Osteoporosis can also be caused by many drugs used in primary care, such as proton pump inhibitors, selective serotonin reuptake inhibitors, glucocorticoids, anticancer drugs, and thiazolidinedione [21]. In addition, various diseases and health conditions can lead to osteoporosis or fractures. To overcome the limitations of previous studies, we excluded several osteoporosis-inducing drug prescription codes and 7 osteoporosislinked disease categories.

Although previous studies have shown a higher prevalence of osteoporosis in patients with IBS [22], the pathogenesis behind this association is not clear. However, a few underlying mechanisms have been proposed. One proposed mechanism is an alteration of serotonin levels due to the mucosal inflammation associated with IBS. Previous studies have reported that gut-derived serotonin reduces osteoblast proliferation by binding to the receptor HTR1B, which is expressed on the osteoblast surface. Subsequent inhibition of the phosphorylation of the cAMP-responsive element-binding protein by phosphokinase A may affect Wnt signaling, leading to bone loss $[23,24]$. Clinical studies have also shown that the use of selective serotonin reuptake inhibitor is associated with an increased risk of osteoporosis and osteoporotic fractures [25]. Although patients with IBS do not display the signs of intestinal inflammation, it has been reported that they have higher levels of cytokines, such as interleukin (IL)-1 $\beta$, tumor necrosis factor, IL-6, and IL-8, which increase the production of serotonin and histamine in association with inflammation. Another possible etiology is the low intake of calcium and vitamin D among patients with IBS. Many patients with IBS tend to avoid dairy products owing to perceived lactose intolerance or because they are following the low-FODMAP (fermentable oligosaccharides, disaccharides, monosaccharides, and polyols) diet [26]. Furthermore, current evidence suggests that low vitamin D levels are common among the IBS population [27]. Vitamin D deficiency increases the serum levels of the parathyroid hormone, leading to high bone turnover, increased bone absorption, subsequent bone loss, and the release of calcium ions into the bloodstream [28]. Calcium and vitamin D are particularly important in the age-related loss of bone density and skeletal muscle mass; however, other minerals, such as magnesium, also have an important role [29]. Additionally, protein may have both beneficial and detrimental effects on bone health depending on various factors, including protein sources. On the other hand, current evidence shows no adverse effects of higher protein intake. Study findings have been heterogeneous, and confounding cannot be excluded; high-quality, longterm studies are needed to clarify the role of dietary protein in bone health [30].

Our study has several key strengths allowing it to overcome the limitations of previous studies. First, few previous studies have described the risk of osteoporosis in patients with IBS; thus, our findings may provide a helpful contribution to the management of these patients. Second, in order to evaluate the risk of osteoporosis more precisely, we compared the IBS group with an age-matched control group. Nevertheless, this study also had several limitations. First, our evidence was derived from an observational cohort study. Osteoporosis often remains undiagnosed and untreated because people usually do not know that they have weak bones until they suffer a fracture. Second, our results cannot be generalized to other ethnic groups because all the subjects in this study were ethnic Koreans. Third, we identified IBS solely based on diagnostic codes, making it possible that some cases were misclassified. Thus, future studies should confirm the diagnosis of IBS via a complete medical history, physical examination, and tests to rule out other conditions. Fourth, we likewise identified osteoporosis solely on the basis of diagnostic codes, instead of performing a diagnostic test, such as DEXA.

\section{Conclusion}

Our data suggest that there may be an increased incidence of osteoporosis and osteoporotic fractures in women with IBS compared to that in age-matched controls. To prevent osteoporosis in patients with IBS, recommendations for proper nutritional intake, such as regarding calcium, vitamin $\mathrm{D}$, magnesium, and protein, are needed.

\section{Acknowledgements}

This work was supported by a clinical research grant from $\mathrm{Pu}$ san National University Hospital in 2020. However, this funding source had no role in the design of this study or in its execution, analyses, data interpretation, or the decision to submit results. 


\section{Statement of Ethics}

The study was approved by the Local Ethics Committee (approval no. E-2014090, July 25, 2014).

\section{Conflict of Interest Statement}

The authors have no conflicts of interest to declare.

\section{Author Contributions}

S.-Y. Lee, H.-R. Hwang, Y.-H. Yi, Y.-J. Kim, J.-M. Kim, Y.-H. Cho, J.-G. Lee, Y.-J. Tak, S.H. Lee, E.J. Park, and Y. Lee contributed to study conception and design; H.-R. Hwang, Y.-H. Yi, S.H. Lee, E.J. Park, and Y. Lee contributed to data analysis and interpretation, and drafting of manuscript; H.-R. Hwang, S.-Y. Lee, and J.-M. Kim contributed data management; H.-R. Hwang, Y.-H. Yi, Y.-J. Kim, S.-Y. Lee, J.-M. Kim, Y.-H. Cho, J.-G. Lee, and Y.-J. Tak contributed to reviewing the manuscript and provided the final approval of the manuscript; and H.-R. Hwang, S.-Y. Lee, and Y.-H. Yi contributed to revising the manuscript and finalizing submission.

\section{References}

1 Houghton LA, Heitkemper M, Crowell M, Emmanuel A, Halpert A, McRoberts JA, et al. Age, gender and women's health and the patient. Gastroenterology. 2016 Feb 15. Epub ahead of print. S0016-5085(16)00183-9.

2 Bengtsson M, Sjöberg K, Candamio M, Lerman A, Ohlsson B. Anxiety in close relationships is higher and self-esteem lower in patients with irritable bowel syndrome compared to patients with inflammatory bowel disease. Eur J Intern Med. 2013 Apr;24(3): 266-72.

3 Lee SY, Lee KJ, Kim SJ, Cho SW. Prevalence and risk factors for overlaps between gastroesophageal reflux disease, dyspepsia, and irritable bowel syndrome: a population-based study. Digestion. 2009;79(3):196-201.

4 Franco CB. Osteoporosis in gastrointestinal diseases. Transl Gastrointest Cancer. 2015 Jan;4(1):57-68.

5 Compston J, Cooper A, Cooper C, Gittoes N, Gregson C, Harvey N, et al. UK clinical guideline for the prevention and treatment of osteoporosis. Arch Osteoporos. 2017 Dec;12(1):43.

6 Lima CA, Lyra AC, Rocha R, Santana GO. Risk factors for osteoporosis in inflammatory bowel disease patients. World J Gastrointest Pathophysiol. 2015 Nov 15;6(4):210-8.

7 Bijelic R, Milicevic S, Balaban J. Risk factors for osteoporosis in postmenopausal women. Med Arch. 2017 Feb;71(1):25-8. Epub 2017 Feb 5.

8 Stobaugh DJ, Deepak P, Ehrenpreis ED. Increased risk of osteoporosis-related fractures in patients with irritable bowel syndrome. Osteoporos Int. 2013 Apr;24(4):1169-75.

9 Yen CM, Muo CH, Lin MC, Chang SN, Chang YJ, Kao $\mathrm{CH}$. A nationwide population cohort study: irritable bowel syndrome is a risk factor for osteoporosis. Eur J Intern Med. 2014 Jan; 25(1):87-91.

10 Ryu D-R. Introduction to the medical research using national health insurance claims database. Ewha Med J. 2017 Apr;40(2):66-70.

11 Tayebati SK, Nittari G, Mahdi SS, Ioannidis N, Sibilio F, Amenta F. Identification of World Health Organisation ship's medicine chest contents by anatomical therapeutic chemical (ATC) classification codes. Int Marit Health. 2017;68(1):39-45.

12 Kim JA, Yoon S, Kim LY, Kim DS. Towards actualizing the value potential of Korea health insurance review and assessment (HIRA) data as a resource for health research: strengths, limitations, applications, and strategies for optimal use of HIRA data. J Korean Med Sci. 2017 May;32(5):718-28.

13 Seong SC, Kim YY, Park SK, Khang YH, Kim $\mathrm{HC}$, Park JH, et al. Cohort profile: the national health insurance service-national health screening cohort (NHIS-HEALS) in Korea. BMJ Open. 2017 Sep 24;7(9):e016640.

14 Mirza F, Canalis E. Secondary osteoporosis: pathophysiology and management. Eur J Endocrinol. 2015 Sep;173(3):R131-51.

15 Oshagbemi OA, Driessen JHM, Pieffers A, Wouters EFM, Geusens P, Vestergaard P, et al. Use of systemic glucocorticoids and the risk of major osteoporotic fractures in patients with sarcoidosis. Osteoporos Int. 2017 Oct;28(10):2859-66.

16 Häussler B, Gothe H, Göl D, Glaeske G, Pientka L, Felsenberg D. Epidemiology, treatment and costs of osteoporosis in Germanythe BoneEVA Study. Osteoporos Int. 2007 Jan;18(1):77-84.

17 Hadji P, Klein S, Häussler B, Kless T, Linder $\mathrm{R}$, Rowinski-Jablokow M, et al. The bone evaluation study (BEST): patient care and persistence to treatment of osteoporosis in Germany. Int J Clin Pharmacol Ther. 2013 Nov; 51(11):868-72

18 Hadji P, Klein S, Gothe H, Häussler B, Kless $\mathrm{T}$, Schmidt $\mathrm{T}$, et al. The epidemiology of osteoporosis - bone evaluation study (BEST): an analysis of routine health insurance data. Dtsch Arztebl Int. 2013 Jan;110(4):52-7.

19 Lee YK, Yoon BH, Koo KH. Epidemiology of osteoporosis and osteoporotic fractures in South Korea. Endocrinol Metab. 2013 Jun; 28(2):90-3

20 Abraham BP, Prasad P, Malaty HM. Vitamin D deficiency and corticosteroid use are risk factors for low bone mineral density in inflammatory bowel disease patients. Dig Dis Sci. 2014 Aug;59(8):1878-84.
21 Mazziotti G, Canalis E, Giustina A. Drug-induced osteoporosis: mechanisms and clinical implications. Am J Med. 2010 Oct;123(10): $877-84$

22 Whitehead WE, Palsson OS, Levy RR, Feld $\mathrm{AD}$, Turner M, Von Korff M. Comorbidity in irritable bowel syndrome. Am J Gastroenterol. 2007 Dec;102(12):2767-76.

23 Barbara G, Cremon C, Carini G, Bellacosa L, Zecchi L, De Giorgio R, et al. The immune system in irritable bowel syndrome. J Neurogastroenterol Motil. 2011 Oct;17(4):34959.

24 Cremon C, Carini G, Wang B, Vasina V, Cogliandro RF, De Giorgio R, et al. Intestinal serotonin release, sensory neuron activation, and abdominal pain in irritable bowel syndrome. Am J Gastroenterol. 2011 Jul;106(7): $1290-8$.

25 Rizzoli R, Cooper C, Reginster JY, Abrahamsen B, Adachi JD, Brandi ML, et al. Antidepressant medications and osteoporosis. Bone. 2012 Sep;51(3):606-13.

26 Vesa TH, Seppo LM, Marteau PR, Sahi T, Korpela R. Role of irritable bowel syndrome in subjective lactose intolerance. Am J Clin Nutr. 1998 Apr;67(4):710-5.

27 Williams CE, Williams EA, Corfe BM. Vitamin D status in irritable bowel syndrome and the impact of supplementation on symptoms: what do we know and what do we need to know? Eur J Clin Nutr. 2018 Oct;72(10): 1358-63.

28 Ilić K, Obradović N, Vujasinović-Stupar N. The relationship among hypertension, antihypertensive medications, and osteoporosis: a narrative review. Calcif Tissue Int. 2013 Mar;92(3):217-27.

29 Erem S, Atfi A, Razzaque MS. Anabolic effects of vitamin $\mathrm{D}$ and magnesium in aging bone. J Steroid Biochem Mol Biol. 2019 Oct;193: 105400.

30 Shams-White MM, Chung M, Du M, Fu Z Insogna KL, Karlsen MC, et al. Dietary protein and bone health: a systematic review and meta-analysis from the National Osteoporosis Foundation. Am J Clin Nutr. 2017 Jun; 105(6):1528-43. 